\title{
A Uniformly Distributed Statistic on a Class of Lattice Paths
}

\author{
David Callan \\ Department of Statistics \\ 1210 W. Dayton St, Madison, WI 53706-1693 \\ callan@stat.wisc.edu
}

Submitted: Nov 13, 2003; Accepted: May 15, 2004; Published: Nov 16, 2004

MR Subject Classifications: 05A15

\begin{abstract}
Let $\mathcal{G}_{n}$ denote the set of lattice paths from $(0,0)$ to $(n, n)$ with steps of the form $(i, j)$ where $i$ and $j$ are nonnegative integers, not both zero. Let $\mathcal{D}_{n}$ denote the set of paths in $\mathcal{G}_{n}$ with steps restricted to $(1,0),(0,1),(1,1)$, the so-called Delannoy paths. Stanley has shown that $\left|\mathcal{G}_{n}\right|=2^{n-1}\left|\mathcal{D}_{n}\right|$ and Sulanke has given a bijective proof. Here we give a simple statistic on $\mathcal{G}_{n}$ that is uniformly distributed over the $2^{n-1}$ subsets of $[n-1]=\{1,2, \ldots, n\}$ and takes the value $[n-1]$ precisely on the Delannoy paths.
\end{abstract}

We consider paths in the lattice plane $\mathbb{Z}^{2}$ with arbitrary nonnegative-integer-coordinate steps, that is, steps in $\mathbb{N} \times \mathbb{N} \backslash\{(0,0)\}$, called general lattice paths. A path can be specified by the sequence of its steps or, depending on where the path is situated in $\mathbb{Z}^{2}$, either by its vertices or by its line segments. Let $\mathcal{G}_{n}$ denote the set of general lattice paths from $(0,0)$ to $(n, n)$, counted by sequence $\underline{\mathrm{A} 052141}$ in the On-Line Encyclopedia of Integer Sequences. Let $\mathcal{D}_{n}$ denote the set of paths in $\mathcal{G}_{n}$ with steps restricted to $(1,0),(0,1),(1,1)$, so-called Delannoy paths. Stanley [2, Ex. 6.16] shows that $\left|\mathcal{G}_{n}\right|=2^{n-1}\left|\mathcal{D}_{n}\right|$ and Sulanke [3] has given a bijective proof. See $[4,5,6]$ for other treatments and generalizations of this problem. Here we give a simple statistic on $\mathcal{G}_{n}$ that is uniformly distributed over the $2^{n-1}$ subsets of $[n-1]$ and takes the value $[n-1]$ precisely on the Delannoy paths.

To present this statistic, the following notions are relevant: a path is balanced if its terminal vertex lies on the line of slope 1 through its initial vertex. A path is subdiagonal if it never rises above the line of slope 1 through its initial vertex, and analogously for superdiagonal. A subpath of a path $\pi$ is of course a subsequence of consecutive steps of $\pi$. Since subpaths that do not start at the origin will arise, the reader should not confuse a 
path's inherent property of being subdiagonal with its placement relative to the diagonal line $y=x$.

For $\pi \in \mathcal{G}_{n}$, consider the interior vertical lines: $x=k, 1 \leq k \leq n-1$. Such a line is active for $\pi$ if it contains a vertex of $\pi$-an active vertex - that (i) lies on the line $y=x$, or (ii) lies strictly below $y=x$ and is the initial vertex of a nonempty balanced subdiagonal subpath of $\pi$, or (iii) lies strictly above $y=x$ and is the terminal vertex of a nonempty balanced superdiagonal subpath of $\pi$. If a line is active for $\pi$ by virtue of (i), no other vertex on the line can meet the conditions of (ii) or (iii). If active by virtue of (ii) or (iii), then all path vertices on the line lie strictly to one side of $y=x$ and only the one closest to $y=x$ is active. In any case, an active line contains a unique active vertex.

Proposition 1. A path $\pi \in \mathcal{G}_{n}$ is Delannoy if and only if all its interior vertical lines are active.

Proof. The "only if" part is clear. For the "if" part, suppose all lines are active for $\pi$. If $\pi$ had a line segment of slope $m$ with $0<m<1$, then there would be an interior vertical line containing no vertex of $\pi$ at all, giving an inactive line. If $\pi$ had a line segment $P Q$ of slope $m$ with $1<m<\infty$, then either $P$ is strictly below $y=x$ making the vertical line through $P$ inactive or $Q$ is strictly above $y=x$ likewise giving an inactive line (or both).

Hence all line segments in $\pi$ have slope 0,1 or $\infty$. But a missing interior lattice point in a segment whose slope is 0 or 1 in $\pi$ would clearly give an inactive line. And a segment of slope $\infty$ and length $\geq 2$ in $\pi$ would contain an interior lattice point $P$ either (i) on $y=x$, (ii) above $y=x$, or (iii) below $y=x$. In case (i), the vertical line through $P$ is inactive. In case (ii), let $L$ denote the ray from $P$ heading northeast. If $L$ contains at least one vertex of the path, the first such vertex determines an inactive line. Otherwise, $\pi$ must cross $L$ on a segment $P Q$ of slope $<1$; all lines strictly between $P$ and $Q$ are inactive and there is at least one such. Similarly, case (iii) gives an inactive line. Hence all line segments in $\pi$ have slope 0,1 or $\infty$ and minimum possible length; in other words, $\pi$ is Delannoy.

The active set for $\pi \in \mathcal{G}_{n}$ is $\{k \in[n-1] \mid x=k$ is active for $\pi\}$. Thus for $\pi \in \mathcal{G}_{n}$, Proposition 1 asserts that its active set is $[n-1]$ iff $\pi$ is Delannoy. Our main result is

Theorem 1. The statistic "active set" on $\mathcal{G}_{n}$ is uniformly distributed over all subsets of $[n-1]$.

We will first prove a similar result for subdiagonal paths. Let $\mathcal{S G}_{n}$ and $\mathcal{S D}_{n}$ denote the set of subdiagonal paths in $\mathcal{G}_{n}$ and $\mathcal{D}_{n}$ respectively. Of course, as for $\mathcal{G}_{n}, \pi \in \mathcal{S G}_{n}$ is Delannoy if and only if all its interior vertical lines are active.

Theorem 2. The statistic "active set" restricted to $\mathcal{S G}_{n}$ is also uniformly distributed over all subsets of $[n-1]$. 
To establish Theorem 2 we will define a map $f$ that takes a path in $\mathcal{S G}_{n}$ together with an active line $x=k$ and produces a path in $\mathcal{S G}_{n}$ in which $x=k$ is not active without disturbing the activity status of other lines: it "deactivates" $k$. The map merely deletes the active vertex for $k$ and adjusts the location of some of its successors along the vertical line they lie on. The map is commutative: given $k, \ell$ active for $\pi \in \mathcal{S G}_{n}$, you get the same result deactivating them in either order. Finally, we show the map is invertible so that we may activate or deactivate lines at will. This yields a bijective correspondence between $\mathcal{S G}_{n}$ and $\mathcal{P}([n-1]) \times \mathcal{S D}_{n}$ via "record the active set for $\pi \in \mathcal{S G}_{n}$ and then activate all of $\pi$ 's inactive lines", and thereby establishes Theorem 2.

It is convenient to use the following abbreviations. For lattice points $X, Y, m_{X Y}$ denotes the slope of the line $X Y$ (possibly $\infty$ ). It is also convenient to assume $m_{X X}=\infty$ $\left(m_{X X}=1\right.$ would work just as well, but not $\left.m_{X X}<1\right)$. Also, $L_{X}$ denotes the line through $X$ of slope 1 .

To define $f$, suppose given a subdiagonal path $\pi$ in $\mathcal{S G}_{n}$ and an active $k$. Locate the active vertex $P$ on $x=k$, its predecessor vertex $A$ and its successor vertex $B$ on $\pi$. There are two cases, illustrated in Figures 1 and 2 below.

Case $\mathbf{m}_{\mathbf{A P}}<1$. Find the first vertex $Q$ on $\pi$ after $P$ that is strictly above $L_{P}$. The existence of $Q$ is guaranteed because $m_{A P}<1$. Lower $\pi$ 's vertices $B$ through (meaning up to and including) the predecessor of $Q$ by $h$ units vertically where $h=$ vertical distance from $P$ down to $B$ (possibly 0 ). Note that the predecessor of $Q$ may be $B$. Finally, delete $P$.

Case $\mathbf{m}_{\mathrm{AP}} \geq \mathbf{1}$ (including $m_{A P}=\infty$ ). Find the first vertex $Q$ on $\pi$ after $P$ that terminates a nonempty balanced subdiagonal subpath starting at $P$. Note that $Q$ may be $B$. Lower $\pi$ 's vertices $B$ through the predecessor of $Q$ on $\pi$ by $h$ units vertically where now $h=$ vertical distance from $P$ down to $L_{A}$ (not to $A$ ). Again $h$ may $=0$ but, unlike Case 1 , even if $h>0$ the set of vertices to be lowered may be vacuous - this occurs if $Q=B$-and then no vertices actually get lowered. Finally, delete $P$.

Figure 1 gives an example of Case $m_{A P}<1$, Figure 2 gives an example of Case $m_{A P} \geq 1$, and Figure 3 gives the action of $f$ on all 6 paths in $\mathcal{S G}_{2}$ for which $x=1$ is active, that is, on $\mathcal{S D}_{2}$. The active line is in red (solid line) and becomes a blue inactive line in the image path. The unaltered vertex $A$ is evident in the image path and the vertex $B$ is readily recovered as the first vertex strictly to the right of the now inactive line. Also marked is the projection $B^{\prime}$ of $B$ on the inactive line; $B^{\prime}$ is key to reversing $f$.

To reverse $f$, we must first distinguish cases in the image path. In fact, we find $m_{A B^{\prime}}<1$ in Case $m_{A P}<1$, and $m_{A B^{\prime}} \geq 1$ (including $A=B^{\prime}$ ) in Case $m_{A P} \geq 1$. Then proceed as follows.

For Case $m_{A B^{\prime}}<1$ :

- Retrieve $Q$ as the first vertex after $A$ on the image strictly above the line $L_{B^{\prime}}$. 
- Retrieve $h$ as the vertical distance from $L_{B^{\prime}}$ down to $Q^{\prime}$ 's predecessor. Raise vertices $B$ through $Q$ by $h$ units.

- Retrieve $P$ as $B^{\prime}$.

For Case $m_{A B^{\prime}} \geq 1$ :

- Retrieve $Q$ as the first vertex strictly after $A$ that lies weakly above $L_{A}$.

- Retrieve $h$ as the vertical distance from $Q$ down to $L_{A}$. Raise vertices $B$ through the predecessor of $Q$ by $h$ units.

- Retrieve $P$ at height $h$ above $L_{A}$ on the inactive line.

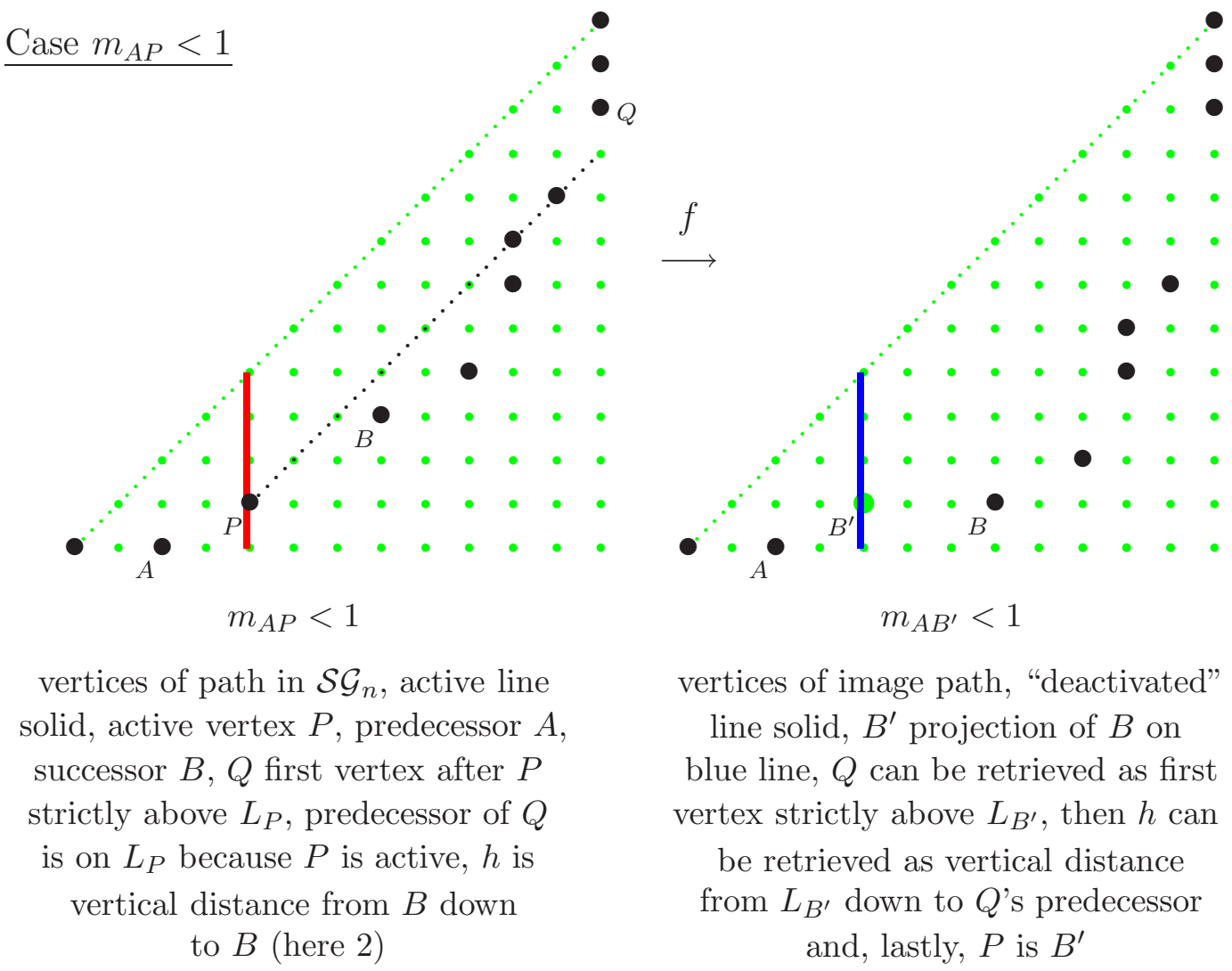

Figure 1 


\section{$\underline{\text { Case } m_{A P} \geq 1}$}

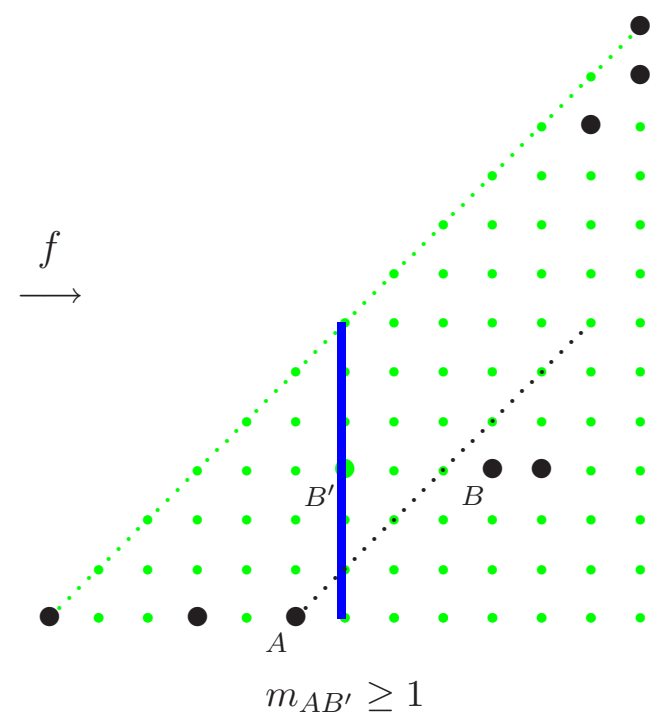

vertices of path in $\mathcal{S G}_{n}$, active line solid, active vertex $P$, predecessor $A$, successor $B, Q$ first vertex on $L_{P}$,

$h$ is vertical distance from $P$ down to $L_{A}$

vertices of image path, "deactivated" line solid, $B^{\prime}$ projection of $B$ on blue line, $Q$ can be retrieved as first vertex weakly above $L_{A}, h$ can be retrieved as vertical distance from $Q$ down to $L_{A}$, and $P$ is $h$ units above $L_{A}$

Figure 2
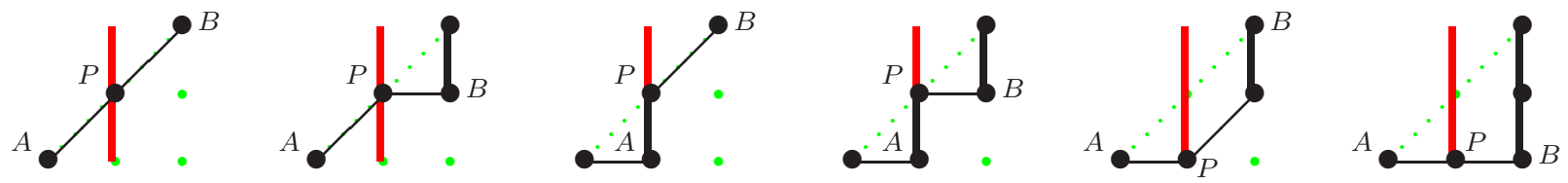

$\downarrow$

$\left.\prod^{\bullet}\right|^{\circ}$
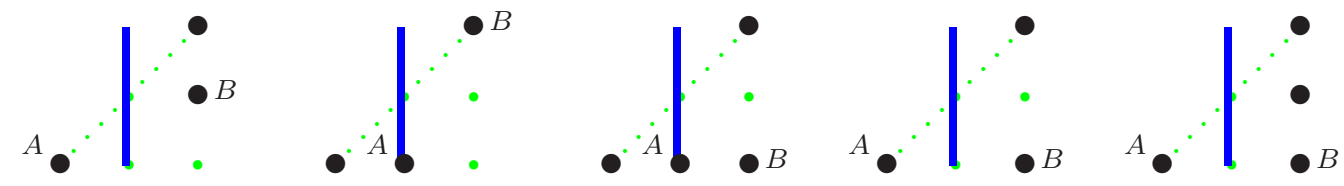

The action of $f$ on $\mathcal{S D}_{2}$

Figure 3 
Having defined the bijection $f:\left\{(\pi, k): \pi \in \mathcal{S G}_{n}, x=k\right.$ active for $\left.\pi\right\} \longrightarrow\{(\pi, k)$ : $\pi \in \mathcal{S G}_{n}, x=k$ inactive for $\left.\pi\right\}$ to establish Theorem 2 , we need only extend $f$ from its definition on $\mathcal{S G}_{n}$ to $\tilde{f}$ defined on $\mathcal{G}_{n}$ : Theorem 1 then follows in the same way as did Theorem 2 .

To do so, given $\pi \in \mathcal{G}_{n}$ and an active $k$, find the active vertex $P$ on $x=k$, its predecessor vertex $A$ and successor vertex $B$. We divide the possibilities into five cases.

Case $1 A, P, B$ all lie weakly below $y=x$. If $m_{A P}<1, \tilde{f}$ coincides with $f$. If $m_{A P} \geq 1$, modify $Q$ in the definition of $f$ : take $Q$ as the first vertex strictly after $P$ that lies weakly above $L_{P}$ rather than "that terminates a nonempty balanced subdiagonal subpath starting at $P$ ". Then $\tilde{f}$ is defined as $f$ was. This modification is necessary because if $P$ lies on $y=x$, there need not be any balanced subdiagonal path $P Q$. To recapture the original path, $h$ is now recaptured as the minimum of the vertical distance from $Q$ down to $L_{A}$ and the vertical distance from $y=x$ down to $L_{A}$.

Case $2 A, P, B$ all lie weakly above $y=x$. Rotate everything $180^{\circ}$ so that Case 1 applies, apply $\tilde{f}$, and rotate back.

Case 3 A strictly above $y=x, P$ strictly below $y=x$. Here $m_{A P}<1$ and $\tilde{f}$ coincides with $f$. Note that $B$ is also strictly below $y=x$ or else $P$ would not be active.

Case $4 P$ strictly above $y=x, B$ strictly below $y=x$. Here $A$, like $P$, is strictly above $y=x$ for the same reason as in Case 3 . Rotate $180^{\circ}$, apply $f$, and rotate back.

Case $5 \quad P$ on $y=x, A, B$ on strictly opposite sides of $y=x$. Here $\tilde{f}$ is simply "delete P".

These five cases are exhaustive and mutually exclusive save for one slight overlap: if $A, P, B$ all lie on $y=x$, then Cases 1 and 2 both apply, but both give the same result, namely, delete $P$.

It is evident from the reversibility of $f$ that, if we know which case an image path arose from, we can recapture the original path. So we need to find distinguishing features in the image paths in the five cases, and to verify that every pair $(\pi, k)$ with $\pi \in \mathcal{G}_{n}$ and $k$ inactive for $\pi$ falls in one of the image cases.

First, we can recover $A, B$ in all cases as the last vertex preceding the lattice point $(k, k)$ and the first vertex following $(k, k)$ respectively, where lattice points are ordered primarily by $x$-coordinate and secondarily by $y$-coordinate. The following Table now gives distinguishing features in the five cases. 


\begin{tabular}{|c|c|c|}
\hline Case & domain path $\pi, x=k$ active & image path $f(\pi), x=k$ inactive \\
\hline \hline 1 & $A, P, B$ all weakly below $y=x$ & $A, B$ weakly below $y=x$ \\
\hline 2 & $A, P, B$ all weakly above $y=x$ & $A, B$ weakly above $y=x$ \\
\hline 3 & $A$ strictly above $y=x$ and & $A$ strictly above $y=x$ and \\
& $P, B$ strictly below $y=x$ & $B$ strictly below $y=k$ \\
\hline 4 & $A, P$ strictly above $y=x$ and & $A$ strictly above $y=k$ and \\
& $B$ strictly below $y=x$ & $B$ strictly below $y=x$ \\
\hline 5 & $P$ on $y=x$ and $A, B$ on strictly & $\begin{array}{c}A, B \text { on strictly opposite sides } \\
\text { of } y=x \text { and } A, B \text { on weakly } \\
\end{array}$ \\
opposite sides of $y=x$ & opposite sides of $y=k$ \\
\hline
\end{tabular}

How to determine the case $\tilde{f}(\pi)$ came from

The image path cases are mutually exclusive save for the overlap in cases 1 and 2 when $A$ and $B$ both lie on $y=x$. Let us confirm they are exhaustive. If $A$ and $B$ lie weakly on the same side of $y=x$, then case 1 or 2 applies. Otherwise, $A$ and $B$ lie on strictly opposite sides of $y=x$. Now, if $A$ is strictly below $y=x$, part of case 5 applies (with $A$ strictly below $y=x, B$ strictly above $y=x$; this forces $A, B$ to lie weakly -in fact, strictly - on opposite sides of $y=k$ ).

This leaves the case $A$ strictly above $y=x$ (for if $A$ were on $y=x$, then $A$ and $B$ would lie weakly on the same side of $y=x$ ) and $B$ strictly below $y=x$. If $A, B$ both lie strictly below $y=k$, Case 3 applies. If $A, B$ both lie strictly above $y=k$, Case 4 applies. The remaining case- $A, B$ weakly on opposite sides of $y=k$-is the other part of Case 5 .

Figure 4 gives an example of $\tilde{f}$ (Case 1 ). 


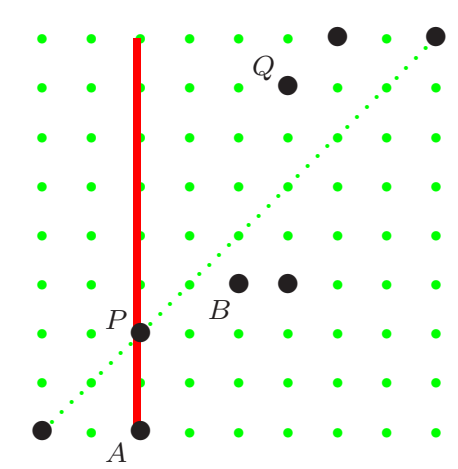

$m_{A P} \geq 1$ and so $h$ is vertical distance from $P$ down to $L_{A}$ (here 2 )

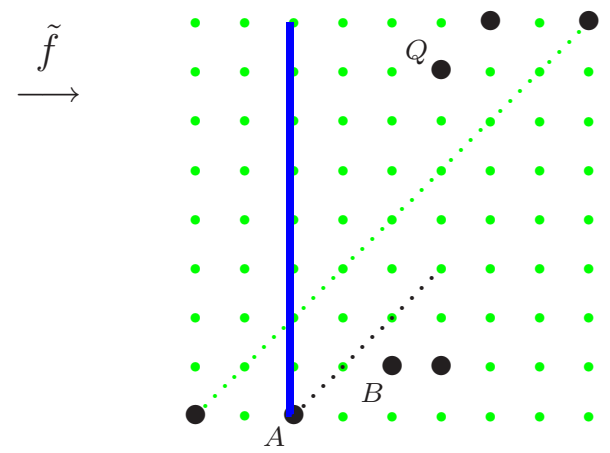

$Q$ can be retrieved as before, $h$ can be retrieved as minimum of vertical distance from $Q$ down to $L_{A}$ (here 4) and vertical distance from $y=x$ down to $L_{A}$ (here 2)

Figure 4

This completes the proof of Theorem 1.

\section{References}

[1] The On-Line Encyclopedia of Integer Sequences.

[2] Richard P. Stanley, Enumerative Combinatorics Vol. 2, Cambridge University Press, 1999.

[3] Robert Sulanke, Counting lattice paths by Narayana polynomials, Electronic Journal of Combinatorics, 7, Art. R40, (2000).

[4] Katherine Humphreys and Heinrich Niederhausen, Counting lattice paths taking steps in infinitely many directions under special access restrictions, Theoretical Computer Science 319, (2004) 385-409.

[5] Robert Sulanke and E.Duchi, The $2^{n-1}$ factor for multi-dimensional lattice paths with diagonal steps, Seminaire Lotharingien de Combinatoire, Art.B51c, 2003.

[6] Robert Sulanke, Generalizing Narayana and Schroder numbers to higher dimensions, Electronic Journal of Combinatorics, 11(1), Art. R54, 2004. 